\title{
Complicated infective endocarditis: a case series
}

Joo Seop Kim', Min-Kyung Kang ${ }^{1 *}$, A. Jin Cho², Yu Bin Seo ${ }^{3}$ and Kun II Kim

\begin{abstract}
Background: Infective endocarditis is associated with not only cardiac complications but also neurologic, renal, musculoskeletal, and systemic complications related to the infection, such as embolization, metastatic infection, and mycotic aneurysm.

Case presentation: We report three cases (the first patient is Chinese and the other two are Koreans) of complicated infective endocarditis; two of the cases were associated with a mycotic aneurysm, and one case was associated with a splenic abscess. One case of a patient with prosthetic valve endocarditis was complicated by intracerebral hemorrhage caused by mycotic aneurysm rupture. A second case of a patient with right-sided valve endocarditis associated with a central catheter was complicated by an abdominal aortic mycotic aneurysm. The third patient had a splenic infarction and abscess associated with infected cardiac thrombi.

Conclusions: Complicated infective endocarditis is rare and is associated with cardiac, neurologic, renal, musculoskeletal, and systemic complications related to infection, such as embolization, metastatic infection, and mycotic aneurysm. Infective endocarditis caused by Staphylococcus aureus is more frequently associated with complications. Because the mortality rate increases when complications develop, aggressive antibiotic therapy and surgery, combined with specific treatments for the complications, are necessary.
\end{abstract}

Keywords: Complications, Infective endocarditis, Mycotic aneurysm

\section{Background}

Infective endocarditis (IE) is associated with not only cardiac complications but also neurologic, renal, musculoskeletal, and systemic complications related to infection, such as embolization, metastatic infection, and mycotic aneurysm (MA) [1]. We report three cases of patients with complicated IE; two were associated with MA, and one was associated with splenic abscess.

\section{Case presentations \\ Patient 1}

A 57-year-old Chinese woman presented to our hospital with generalized weakness. Her medical history included hypertension and early liver cirrhosis caused by chronic viral hepatitis C (platelet count 104,000/ $\mu \mathrm{l}$ ). She had undergone mitral valve replacement (MVR) with a Hancock II 27-mm prosthesis (Medtronic, Minneapolis,

\footnotetext{
* Correspondence: homes78@naver.com

'Division of Cardiology, Kangnam Sacred Heart Hospital, Hallym University

Medical Center, Seoul, South Korea

Full list of author information is available at the end of the article
}

MN, USA) for mitral valve prolapse 1 month earlier. Her blood pressure was $100 / 60 \mathrm{mmHg}$, her breathing rate was 12 breaths/minute, her heart rate was 121 beats/minute, and her body temperature was $36.5{ }^{\circ} \mathrm{C}$. She appeared acutely ill and was dehydrated. The result of an initial chest $x$-ray was normal, and the patient's electrocardiogram showed sinus tachycardia. Transthoracic echocardiography (TTE) showed normal prosthetic valve motion without evidence of vegetation or paravalvular leakage, but the patient's mean diastolic pressure gradient was elevated at $10.3 \mathrm{mmHg}$ (Fig. 1a) [2]. She had been discharged on warfarin and other medications after successful MVR without complications. Transesophageal echocardiography (TEE) (Fig. 1b and Additional file 1: Video 1) showed hyperdynamic echogenic material attached to the prosthetic MV. During preparation for redo surgery with administration of appropriate antibiotics, the patient suddenly had a generalized seizure with decreased mentation. Brain computed tomography $(\mathrm{CT})$ and magnetic resonance imaging 

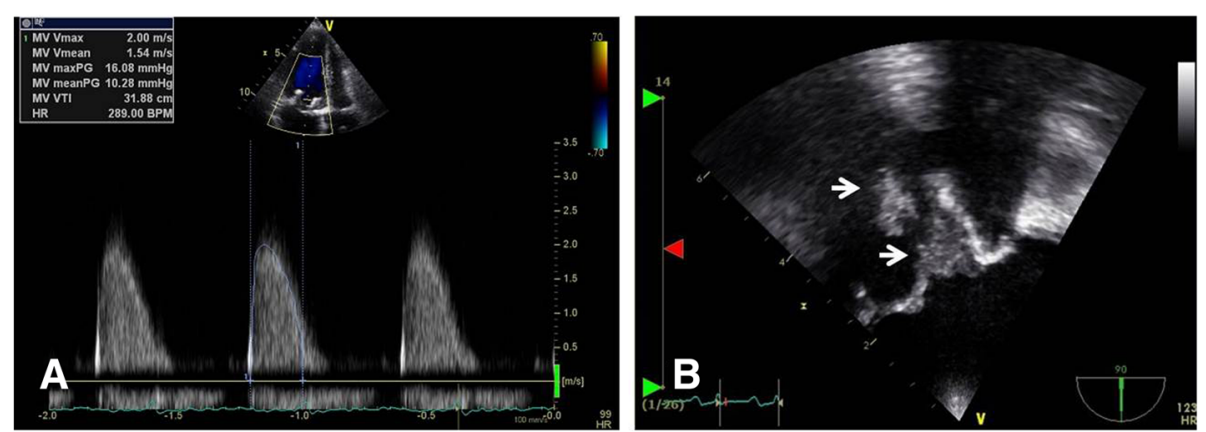

Fig. 1 Transthoracic echocardiograms showing an elevated mean diastolic pressure gradient (a) of $10.3 \mathrm{mmHg}$ and a hyperdynamic echogenic mass attached to the prosthetic MV (b) (white arrows)

(Fig. 2a, b) revealed acute hemorrhage with perilesional edema in the bilateral cerebellum causing obstructive hydrocephalus, suggestive of MA rupture. She was transferred to the intensive care unit with an indwelling external ventricular drain (EVD) for monitoring. We performed a redo MVR with a Hancock II 27-mm prosthesis after resolution of her hemorrhage.

\section{Patient 2}

A 70-year-old Korean woman presented with generalized weakness and headache. Her medical history included diabetes mellitus and hypertension. Her physical examination revealed her blood pressure was 163/81 $\mathrm{mmHg}$ and her pulse rate was 91 beats/minute. Brain CT revealed a chronic left subdural frontotemporal hemorrhage. After burr hole trephination was performed, generalized edema developed because of acute kidney injury (creatinine level $0.86 \rightarrow 1.89 \mathrm{mg} / \mathrm{dl})$. A peripherally inserted central catheter (PICC) was placed. The patient developed a fever $\left(38.1{ }^{\circ} \mathrm{C}\right)$ after 3 weeks, without a definite source of infection. TEE revealed a globular, mobile, echogenic mass $(1.91 \times 1.0 \mathrm{~cm})($ Fig. 3a, b) attached to the tricuspid valve.
Blood cultures revealed Staphylococcus aureus sensitive to vancomycin. The patient's fever subsided after treatment with antibiotics, but a vegetation and persistent septicemia were noted after 2 weeks. We performed coronary angiography prior to possible valve surgery and observed no significant coronary obstruction, but a large saccular aneurysm was detected in the infrarenal abdominal aorta (Fig. 4a and Additional file 2: Video 2). CT indicated this was a newly developed abdominal aortic aneurysm (maximum diameter $5.2 \mathrm{~cm}$ ) (Fig. 4b, c) that had not been present 2 years previously (Fig. 4d). The appearance was suggestive of an MA associated with IE. We recommended valve surgery and endovascular stenting or surgical removal of the MA in sequence, but the patient's guardians refused. The patient was discharged to a private convalescent hospital and was lost to followup. We suspect IE developed in association with PICC placement and that persistent septicemia, despite use of proper antibiotics, led to an MA.

\section{Patient 3}

A 76-year-old Korean woman with acute onset of flank pain caused by splenic infarction and abscess was

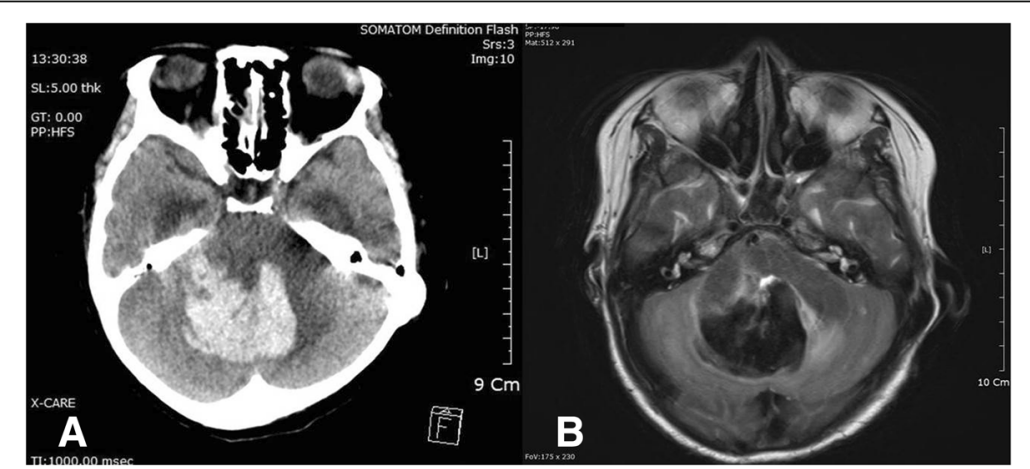

Fig. 2 Brain computed tomography (a) and magnetic resonance imaging (b) revealing acute hemorrhage with perilesional edema in the bilateral cerebellum causing obstructive hydrocephalus, suggestive of mycotic aneurysmal rupture 


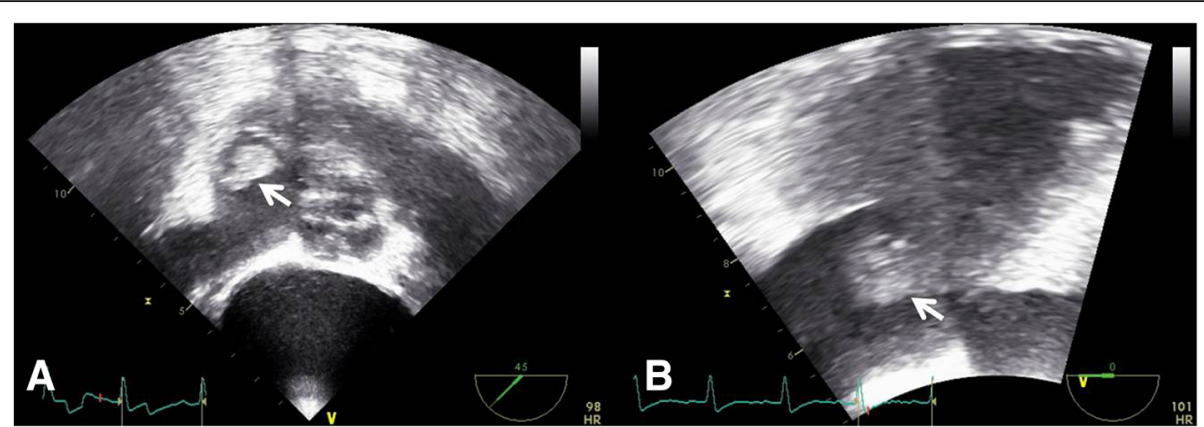

Fig. 3 a and $\mathbf{b}$ Transesophageal echocardiography revealing a globular, mobile, echogenic mass $(1.91 \times 1.0 \mathrm{~cm}$; arrows $)$ attached to the tricuspid valve

transferred to our hospital with percutaneous drainage (Fig. 5). She had been diagnosed with a stroke in our hospital 3 months earlier. Other than fever $\left(38.1{ }^{\circ} \mathrm{C}\right)$, she had stable vital signs. Although splenic infarction or embolism is common, splenic abscess is rare. In every patient diagnosed with splenic infarction, a search for the possible source of emboli should be performed, and IE is the most common cause [3]. There was no evidence of IE in this patient, but slightly increased mitral regurgitation (grade 1-2) (Additional file 3: Video 3 and Additional file 4: Video 4) was noted by TTE. TEE revealed a thickened, nonhomogeneous area with an echo-dense appearance around the aortic root (Fig. 6a) and discontinuous endocardial tissue (Fig. 6b) with flow communication detected by color and pulsed wave Doppler ultrasound (Fig. 6c, d). This patient needed surgery for locally uncontrolled infection [4]. A weblike structure with interruption of endocardial tissue continuity was noted (Fig. 7a), and thrombi were observed within the pocket (Fig. 7b). The patient recovered fully and was discharged.

\section{Discussion}

IE is associated with cardiac, neurologic, renal, and musculoskeletal complications. Predisposing factors include the infecting pathogen, duration of illness prior to therapy, and underlying comorbidities [1]. IE caused by $S$. aureus is associated with complications more frequently than other pathogens [5].

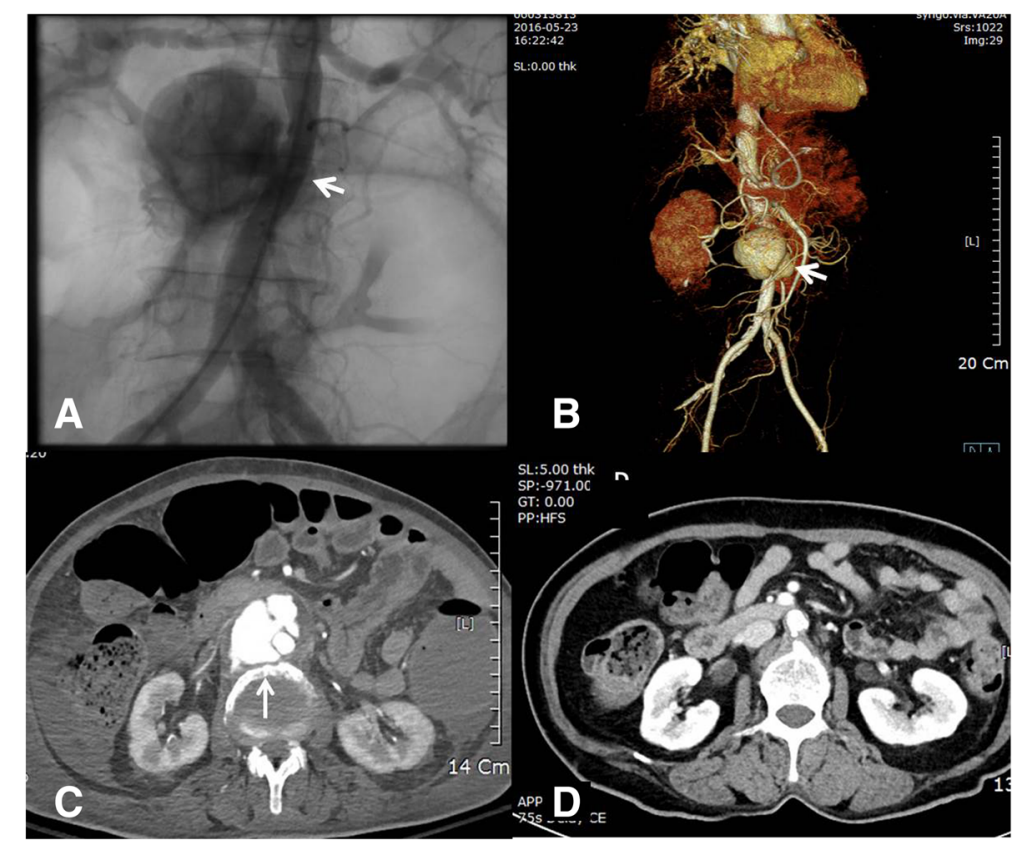

Fig. 4 A large saccular aneurysm was detected in the infrarenal abdominal aorta by aortography (a, white arrow) and computed tomography (b and $\mathbf{c}$, white arrows). The aneurysm had not been present 2 years previously (d) 

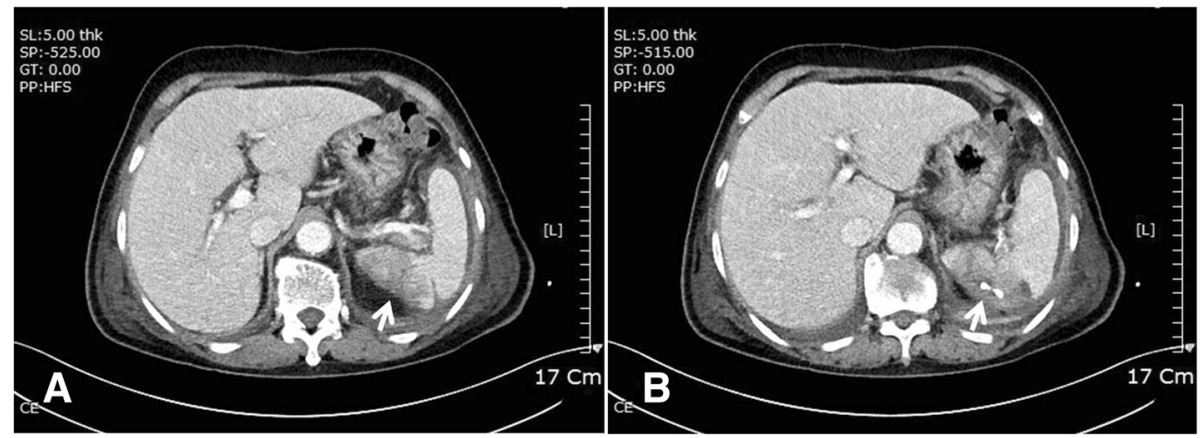

Fig. 5 Computed tomography shows splenic infarction (a, white arrow) and abscess with percutaneous drainage (b, white arrow)

MA can develop in the cerebral or systemic circulation in the setting of IE [6], and cerebral hemorrhage caused by stroke or a ruptured MA can cause neurologic complications. Direct bacterial inoculation, bacteremic seeding, contiguous infection, and septic emboli are the sources of MA. Intracranial MA usually involves more distal portions of the middle cerebral artery, as in patient 1 , and unruptured aneurysms may be managed with antibiotics alone. However, ruptured aneurysms should be managed with a combination of antibiotics and surgery [6]. Patient 1 developed intracranial hemorrhage and a seizure; we initially attempted conservative treatment with EVD and close monitoring in the intensive care unit, and then we performed a redo MVR after stabilization [4]. The perioperative mortality rate for infected aortic aneurysms is
$15 \%$ to $20 \%$ [7]. Survival is better for an infrarenal abdominal aortic aneurysms than for noninfrarenal aneurysms ( $96 \%$ versus $57 \%$, respectively) [8]. Therefore, we suggest debridement of an infected infrarenal aortic aneurysm, along with extraanatomic reconstruction. The guardians of patient 2 refused all surgery because of the high morbidity and mortality risk.

In contrast to relatively common splenic infarctions in patients with embolic events, splenic abscesses are very rare and fatal complications of IE [3]. The treatments of choice are antibiotics, splenectomy, and valve replacement surgery. After patient 3 was stabilized with drainage of a splenic abscess, we decided to perform valve replacement surgery and debridement of the perforated intracardiac abscess pocket. The aortic and mitral valves

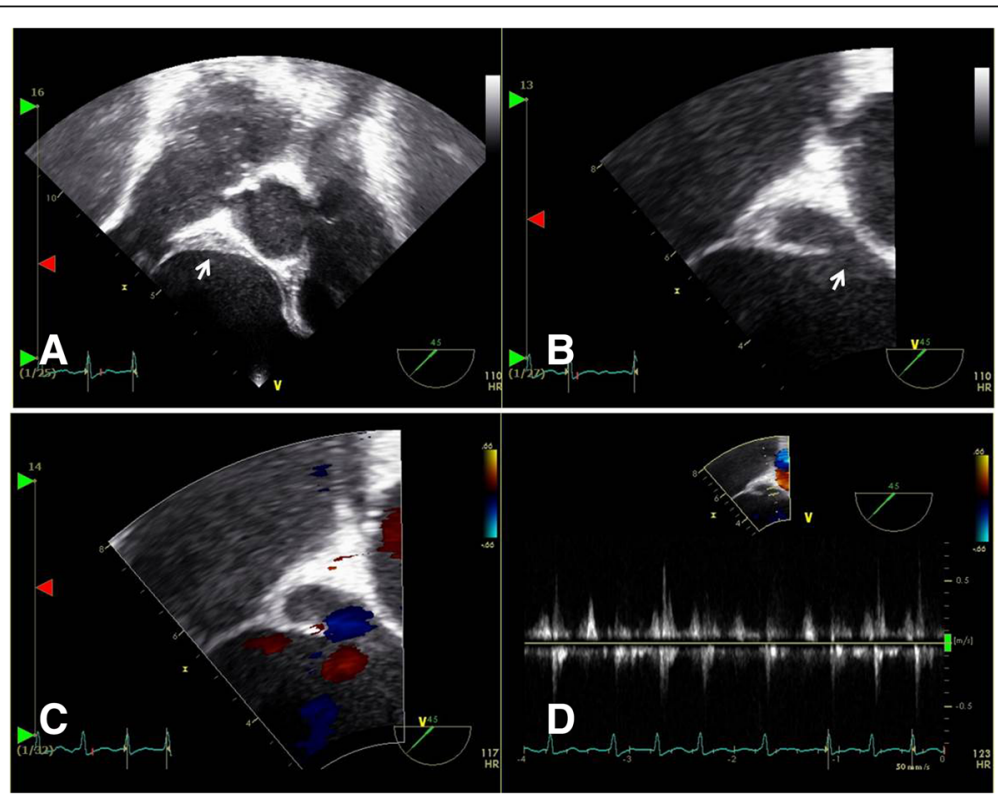

Fig. 6 Transesophageal echocardiography shows a thickened nonhomogeneous area with echo-dense appearance around the aortic root (a, white arrow), as well as discontinuous endocardial tissue (b, white arrow) with flow communication detected by color and pulsed wave Doppler ultrasound (c and $\mathbf{d}$ ) 


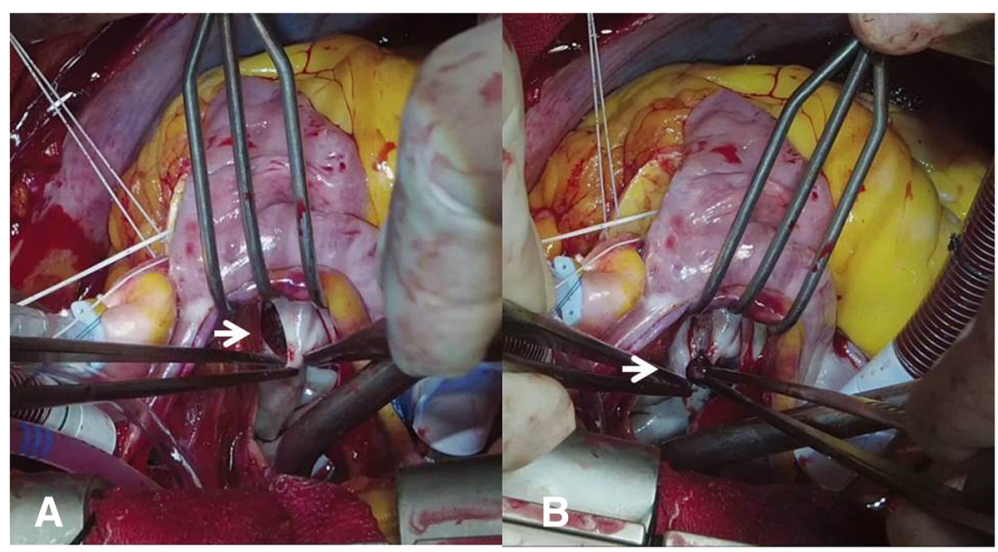

Fig. 7 Intraoperative views show a weblike structure with interruption of endocardial tissue continuity (a, white arrow) and that thrombi were present in the pocket (b, white arrow)

were relatively clean, except for degenerative changes, but infected thrombi were noted in a phlegmon.

\section{Conclusions}

IE is associated with cardiac, neurologic, renal, musculoskeletal, and systemic complications related to the infection (embolization, metastatic infection, and MA). Predisposing factors include the infecting pathogen, duration of illness, prior therapy, and underlying comorbidities. Complications can occur before, during, and after completion of therapy. IE caused by $S$. aureus is associated with complications more frequently. Because the mortality rate increases with complications, aggressive antibiotic therapy combined with surgery and other specific treatments for complications is necessary.

\section{Additional files}

Additional file 1: TEE showed a hyper-dynamic echogenic mass attached to the prosthetic mitral valve. (WMV $804 \mathrm{~kb}$ )

Additional file 2: A large saccular aneurysm was detected in the infrarenal abdominal aorta by aortography. (WMV $2144 \mathrm{~kb}$ )

Additional file 3: TTE showed mild mitral regurgitation. (WMV $1254 \mathrm{~kb}$ )

Additional file 4: TTE showed increased mitral regurgitation. (WMV 1692 kb)

\section{Abbreviations}

CT: Computed tomography; EVD: External ventricular drain; IE: Infective endocarditis; MA: Mycotic aneurysm; MVR: Mitral valve replacement; PICC: Peripherally inserted central catheter; TEE: Transesophageal echocardiography; TTE: Transthoracic echocardiography

\section{Funding}

There was no funding for this case report.

\section{Availability of data and materials}

The datasets used and/or analyzed during the present study are available from the corresponding author on reasonable request.

\section{Authors' contributions}

All authors contributed to the conception of this case report, the literature review, the analysis and interpretation of the material, and drafting and critical revision of the manuscript; All authors agree to be accountable for all aspects of the work in ensuring that questions related to the accuracy or integrity of any part of the work are appropriately investigated and resolved. All authors read and approved the final manuscript.

\section{Competing interests}

The authors declare that they have no competing interests.

\section{Consent for publication}

Written informed consent was obtained from the patients for publication of this case report and any accompanying images. A copy of the written consent is available for review by the Editor-in-Chief of this journal.

Ethics approval and consent to participate

Not applicable.

\section{Publisher's Note}

Springer Nature remains neutral with regard to jurisdictional claims in published maps and institutional affiliations.

\section{Author details}

'Division of Cardiology, Kangnam Sacred Heart Hospital, Hallym University Medical Center, Seoul, South Korea. ${ }^{2}$ Division of Nephrology, Kangnam Sacred Heart Hospital, Hallym University Medical Center, Seoul, South Korea. ${ }^{3}$ Division of Infection, Kangnam Sacred Heart Hospital, Hallym University Medical Center, Seoul, South Korea. ${ }^{4}$ Division of Cardiothoracic Surgery, Kangnam Sacred Heart Hospital, Hallym University Medical Center, Seoul, South Korea.

Received: 17 January 2017 Accepted: 23 March 2017 Published online: 08 May 2017

\section{References}

1. Hoen B, Duval X. Infective endocarditis. N Engl J Med. 2013;368:1425-33.

2. Namboodiri N, Shajeem O, Tharakan JA, Sankarkumar R, Titus T, Valaparambil A, et al. Doppler echocardiographic assessment of TTK Chitra prosthetic heart valve in the mitral position. Eur J Echocardiogr. 2008;9:599-604.

3. Wang CC, Lee CH, Chan CY, Chen HW. Splenic infarction and abscess complicating infective endocarditis. Am J Emerg Med. 2009;27:1021. e3-5.

4. Habib G, Lancellotti P, Antunes MJ, Bongiorni MG, Casalta JP, Del Zotti F, et al. 2015 ESC guidelines for the management of infective endocarditis: the Task Force for the Management of Infective Endocarditis of the European Society of Cardiology (ESC). Eur Heart J. 2015;36:3075-128.

5. Fowler Jr VG, Miro JM, Hoen B, Cabell CH, Abrutyn E, Rubinstein E, et al. Staphylococcus aureus endocarditis: a consequence of medical progress. JAMA. 2005;293:3012-21.

6. Bisdas T, Teebken OE. Mycotic or infected aneurysm? Time to change the term. Eur J Vasc Endovasc Surg. 2011;41:570-1. 
7. Oderich GS, Panneton JM, Bower TC, Cherry Jr KJ, Rowland CM, Noel AA, et al. Infected aortic aneurysms: aggressive presentation, complicated early outcome, but durable results. J Vasc Surg. 2001;34:900-8.

8. Lee $\mathrm{CH}$, Hsieh HC, Ko PJ, Li HJ, Kao TC, Yu SY. In situ versus extra-anatomic reconstruction for primary infected infrarenal abdominal aortic aneurysms. J Vasc Surg. 2011;54:64-70

Submit your next manuscript to BioMed Central and we will help you at every step:

- We accept pre-submission inquiries

- Our selector tool helps you to find the most relevant journal

- We provide round the clock customer support

- Convenient online submission

- Thorough peer review

- Inclusion in PubMed and all major indexing services

- Maximum visibility for your research

Submit your manuscript at www.biomedcentral.com/submit 